(3)

Volume 21, 2018

\title{
INFORMING ON A RUGGED LANDSCAPE: How COMPLEXITY DRIVES OUR PREFERRED INFORMATION SOURCES
}

T. Grandon Gill*

Matthew Mullarkey

Ronald K Satterfield

* Corresponding author
University of South Florida, Tampa, FL, USA

University of South Florida, Tampa, FL, USA

University of South Florida, Tampa, FL, USA grandon@usf.edu

mmullarkey@usf.edu

rsatterf@,usf.edu

\section{ABSTRACT}

Aim/Purpose

Provides a theoretical model as to where we should source our information as the environment becomes more complex.

Background

Methodology

Contribution

Findings

Recommendations for Practitioners

Recommendation for Researchers

Impact on Society

Future Research

Develops a theoretical model built on extrinsic complexity and offers a conceptual scheme relating to the relative value of different sources.

The paper is purely conceptual in nature.

Develops a model that could be tested relating to where clients should search for information.

Arguments can be made that different environments warrant different priorities for informing sources.

Assess how your sources of information match your perceived environment.

Consider developing research designs to test the proposed model.

Offers a new way of thinking about informing sources.

Develop propositions from the model that could be empirically tested in future research.

Accepting Editor Eli Cohen | Received: December 17, 2017| Revised: February 3, 2018 | Accepted: February 19, 2018.

Cite as: Gill, T. G., Mullarkey, M., \& Satterfield, R. K. (2018). Informing on a rugged landscape: How complexity drives our preferred information sources. Informing Science: the International Journal of an Emerging Transdiscipline, 21, 1-18. https://doi.org/10.28945/3977

(CC BY-NC 4.0) This article is licensed to you under a Creative Commons Attribution-NonCommercial 4.0 International License. When you copy and redistribute this paper in full or in part, you need to provide proper attribution to it to ensure that others can later locate this work (and to ensure that others do not accuse you of plagiarism). You may (and we encourage you to) adapt, remix, transform, and build upon the material for any non-commercial purposes. This license does not permit you to use this material for commercial purposes. 
Keywords extrinsic complexity, environment, informing sources, client, informer

\section{INTRODUCTION}

From whom should a client seek information in a complex environment? Since the inception of the informing science field (Cohen, 1997, 1999) and others, the informing system has been used as a key building block to understand informing processes. When modeling an informing system, we normally define one or more clients, one or more informers, and one or more channels through which informing takes place (Gill, 2015). Up to this point, however, we have spent very little time looking at two questions related to these systems:

1. When clients are initially constructing such a system, how are the most appropriate informers selected?

2. When an informing system involves multiple informers, how should a client prioritize the degree of attention paid to each source?

These are obviously very broad questions unlikely to have general answers. We believe, however, that the nature of the environment faced by the client proves to be an important factor in determining the most appropriate sources of information. In this paper, we look at one environmental characteristic — extrinsic complexity (Gill \& Mullarkey, 2017) —and develop a theoretical model of how this characteristic might guide clients towards general categories of informers.

We begin with a brief review of extrinsic complexity, a form of complexity closely related to the notion of a fitness landscape from evolutionary biology (Kauffman, 1993). We then review how extrinsic complexity can be framed in the context of informing. We propose two dimensions, survivability and familiarity, to characterize complex environments. We classify informers as to being external vs. internal, and direct vs. indirect. From these definitions, we develop a model to predict how the nature of the environment will drive the choice of informing sources. We then conclude by pointing out the dangers of over-reliance on a particular source, emphasizing the benefits of keeping a variety of informing channels open despite prioritizing those sources that fit with a particular environment.

\section{EXTRINSIC COMPLEXITY AND FITNESS}

Extrinsic complexity is complexity that derives from the nature of the environment facing an agent, the term we will use for a generic entity operating in a particular environment. For our purposes it can be viewed as the amount of information needed to describe the relationship between fitnesssome desired (or desirable) outcome — and each possible state an agent can occupy. A state, in turn, is a description of an agent's position through a set of attributes or variables. The entire mapping between all possible attribute combinations and their associated fitness is referred to as a fitness landscape.

Where extrinsic complexity is low, each attribute that determines the state independently contributes to fitness. Like questions on a multiple-choice test, each attribute has a "best" value to increase fitness that is independent of the values of the other attributes. Where extrinsic complexity is high each attribute's contribution to fitness depends on the values of the other attributes. Like the ingredients in a recipe, whether it is beneficial to include a particular ingredient depends upon what other ingredients are present. Garlic is likely to increase the fitness of a recipe whose main ingredient is lamb; generally speaking, adding garlic is going to work in the opposite direction when added to most desert recipes according to most people's tastes. High extrinsic complexity landscapes are also referred to as rugged landscapes, reflecting the fact that small changes to state can produce large changes to fitness. Returning back to the recipe example, if you omit the half-teaspoon of baking powder from a cake recipe that requires it, the resulting "cake" is likely to resemble construction material-having failed to rise. Yet baking powder represents only a tiny fraction of the entire recipe for the cake. 
Gill, Mullarkey, \& Satterfield

\section{SET FITNESS}

One drawback of fitness landscapes, particularly when applied to living agents (such as you or I) is that an individual state tends to be very specific. For example, the reader's "knowledge state" would necessarily contain attributes describing everything he or she knows. Most likely, it would also require attributes reflecting the path that was taken to get to that knowledge state. To get around this, we introduce the concept of a state-set, a collection of states related to each other by some common attributes. When we refer to Stradivarius violins, for example, we would be identifying a state-set consisting of all violins made by Stradivarius, a subset of the broader set of all violins.

The use of state-sets allows us to address an area of vagueness present in much exiting fitness research. Typically, earlier research assumes that (1) fitness is an ordinal value whose relationship to the biological notion of survival is limited at best, (2) the relationship between attributes and fitness is relatively stable over time, and (3) agents can immediately assess the fitness of a state once they occupy it. Using state-sets, a much tighter linkage between fitness and survival/reproduction can be established (Gill \& Mullarkey, 2017). We refer to this revised definition as set-fitness while discussing fitness assumed by prior rugged fitness landscape models as state-fitness. The set-fitness construct simply looks at the number of agents or entities occupying a particular state-set over time. These sets may be closed, meaning that no new agents are allowed during the period, or open to agents joining the set. For example, the state-set of Stradivarius violins became closed after the master died. The state set of an individual's descendants remains open unless that individual has no children or his/her last living descendant dies. The realized fitness of a state-set between two periods is simply the ratio of ending occupancy to beginning occupancy. A value of 1.0 indicates the count of agents is unchanged between periods, less than 1.0 means occupancy has fallen, greater than 1.0 means that new agents have entered the set. By their very nature, closed sets will have a realized fitness of 1.0 or less; values less than one meaning that agents have dropped out of the set (e.g., left the landscape, failed to survive).

Defining realized fitness with respect to a state-set, rather than a single state, offers advantages in the context of strategy. First, it can be readily used to answer questions such as: "What is the realized fitness of a particular strategy over a given period?" This would involve creating a set of all states meeting the criteria of that strategy and measuring their occupancy at the beginning and end of the period. Second, it provides a mechanism for handling imperfect fit. The counts used to determine occupancy can be weighted, for example, to get a more accurate picture of the overall fitness of a particular industry. A firm's contribution to the count might be weighted by sales. Fractional set membership can also be specified - a technique employed in the artificial intelligence domain known as fuzzy logic. In other words, an organization that meets only some of the characteristics of a strategy whose fitness is being evaluated might be treated as having fractional membership in the set. This fractional membership approach might be employed to accommodate situations such as IBM's rapid strategic shift from product sales to services (without entirely abandoning the former) in the early 1990s.

In the set-fitness conceptual scheme, fitness is defined as the expected value of realized fitness, as illustrated in Figure 1. Where this differs from state-fitness used in existing rugged landscape models is in the assumption that various scenarios could potentially influence realized fitness. Each scenario would come with its own probability, and these probabilities would also be arguments of the fitness function. The outcomes of these scenarios, leading to a realized fitness value, then feed back into the fitness function for the subsequent period. Through this mechanism, events in a particular periodincluding the movement of other agents on the landscape - can exert a significant impact on fitness in subsequent periods. Figure 1 also includes grey swan and black swan scenarios (Taleb, 2007) that can dramatically influence realized fitness that cannot be predicted. This implies set-fitness is unknowable. This assertion holds even in the unlikely event that we can accurately identify all possible predictable scenarios and assess their respective probabilities for a particular period. We can estimate fitness; we cannot observe or determine it. Unfortunately, the very essence of black swan events is that their 
likelihood cannot be reliably estimated. Thus, even our estimates of the possible errors associated with a fitness function will necessarily involve a certain amount of guesswork.

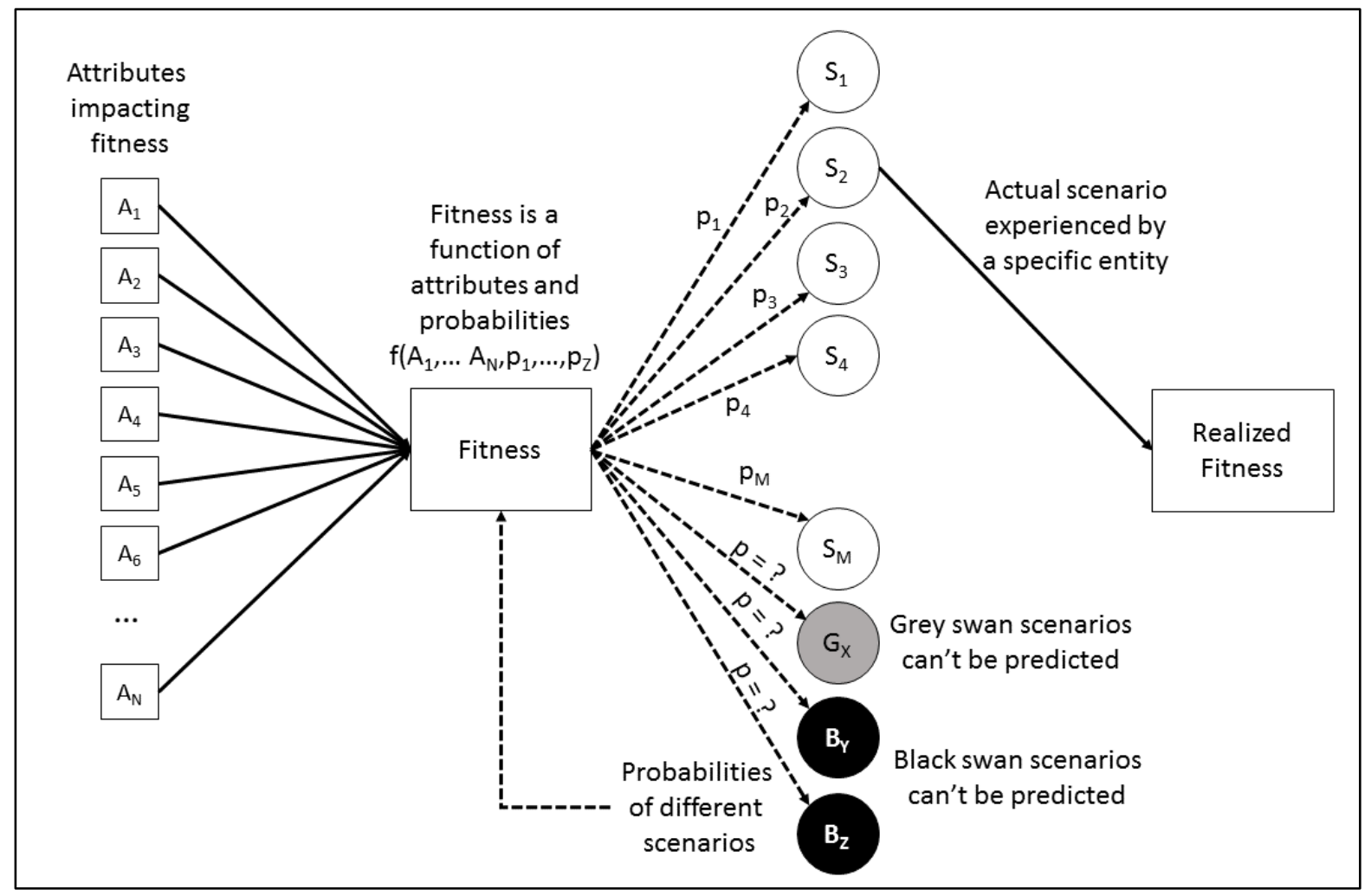

Figure 1: Fitness and Realized Fitness (adapted from Gill \& Mullarkey, 2017)

\section{IMPLICATIONS OF THE SET-FITNESS DEFINITION}

There are three important implications of incorporating set-fitness into rugged landscape models. We consider each in turn.

Dynamic fitness. Most rugged landscape models assume fitness to be static. Under this assumption, the average fitness of a particular state-set does not change over time. Stated another way, a particular fitness landscape is not impacted by the behavior of agents on that landscape. In contrast, set-fitness is inherently dynamic. Entities that transition into and out of a state-set will necessarily impact realized fitness (as a matter for definition). When a particular clothing style becomes popular, the number of individuals choosing to adopt that style will increase from period-to-period (fitness $>1.0$ ). As the fashion leaders discover their innovation becoming commonplace, they may adopt a new style and others may follow. As a result, the fitness of the original clothing style declines in popularity from period-to-period (fitness $<1.0$ ). What is significant here is that is the level of occupancy of a particular state-set that drives its fitness-not necessarily changes to some underlying measure of "goodness" that is intrinsic to the style itself.

Moreover, that impact of occupancy on fitness often falls into predictable patterns. For example, if agents enter a state-set following a standard diffusion S curve (Rogers, 2003), fitness will rise as early adopters pile in and then fall as the state-set reaches saturation. Moreover, occupancy can impact fitness in either direction. In some cases, occupancy could beget more occupancy until a threshold is reached. This would apply to landscapes exhibiting the network effect (Uzzi, 1996, p. 694), whereby increasing occupancy of a state increases its attractiveness (e.g., the more people using email, the more attractive it becomes to non-users). But as occupancy starts to exceed the capacity of a state-set 
some or all existing occupants will leave. Not only will such positive feedback necessarily impact system behavior (e.g., Merali \& Allen, 2011, p. 34), it also introduces an important social dimension to the process.

Unknowability of fitness. Perhaps the most significant difference between the definition we present here and most existing models is the assumption that fitness is both uncertain and unknowable. Although the history of past realized fitness for a particular state-set can offer some useful insights, it may not help an organization. For example, the probability that an organization with stable fitness ratio of 0.95 will exhibit the same survival pattern over 10 periods as one with a realized fitness ratio of 1.0 is roughly $60 \%\left(0.95^{10}\right)$. Effectively, small differences in fitness-differences that can be very important in the long run-are nearly impossible to estimate based on short-term history. Furthermore, when a typical organization that has not experienced major reorganizations or divestitures looks at its own history for insights, its past realized fitness will consist of a series of 1.0 values for all of its past periods. Taleb (2007) specifically cautions the Thanksgiving turkey against predicting future fitness based on past experience...come early November. To the inexperienced turkey, Thanksgiving is the ultimate black swan event.

As per the example just given, we would expect the unknowability of set-fitness to be particularly pronounced at levels near one since occupancy will be relatively stable from period to period. Where the fitness levels of most states in a landscape are substantially lower, actual fitness would be more quickly reflected in survival rates. Thus in classifying landscapes, it can be useful to define a survivability construct, ranging from bostile (substantially less than 1 , and as low as 0 ) to bountiful (at or near 1), that reflects the period-to-period fitness of a closed set of entities. We would expect this construct to change over time since a bountiful landscape is likely to attract entities-creating increased competition for resources or, in the opposite direction, network effects-unless barriers to entry are high. In this regard, the survivability construct can be viewed as a close parallel to the industry attractiveness dependent variable of the five forces model (Porter, 2008). We would further expect that regardless of "average" survivability, there will always be some entities occupying localized states-sets with fitness near 1.0 within the broader industry state-set. Where this is not the case, the industry state-set will eventually become empty of entities (e.g., the mass-produced steam-powered automobile industry).

Adjacency of states. As noted earlier, there are some existing models that expressly consider the potential impact of long-jumps into regions of unknown fitness as a means of contrasting exploration with exploitation (Billinger, Stieglitz, \& Schumacher, 2014). The long-jump vs. adjacent transition dichotomy raises some conceptual issues, however. If a single transition can take an entity from a peak to a distant part of the landscape, then we would argue that the distant state is not-in factdistant. Instead, it is better characterized as an adjacent state of unknown fitness. If that state happens to be of higher fitness (as we might hope it would be, given our decision to undertake the transition), then we were not originally on a peak at all.

In real-world situations, an agent's decision to explore is not typically a blind leap. Rather it is a decision to take incremental steps away from the existing peak, through an intervening valley, towards a new state of potentially higher but uncertain fitness. This is illustrated in Figure 2 from the perspective of an organizational agent. It is unrealistic to assume an organization planning to undertake a radical strategic change from an existing peak would do so without taking a series of preparatory steps prior to the actual transition. These steps would, presumably, impose costs whose associated benefits would not be realized until and unless the transition was completed. For example, when Elon Musk (a co-founder of PayPal) decided to initiate very different ventures, such as Tesla and SpaceX, a direct jump was not possible. Instead, the transition consisted of a series of companybuilding activities that were focused on creating an outcome whose fitness could not be predicted with any accuracy. Presumably, if he had decided to abandon the projects during any of the intermediate states, he would have been worse off than when he started. And, even today, the long term fitness of the new organizations is far from guaranteed. 


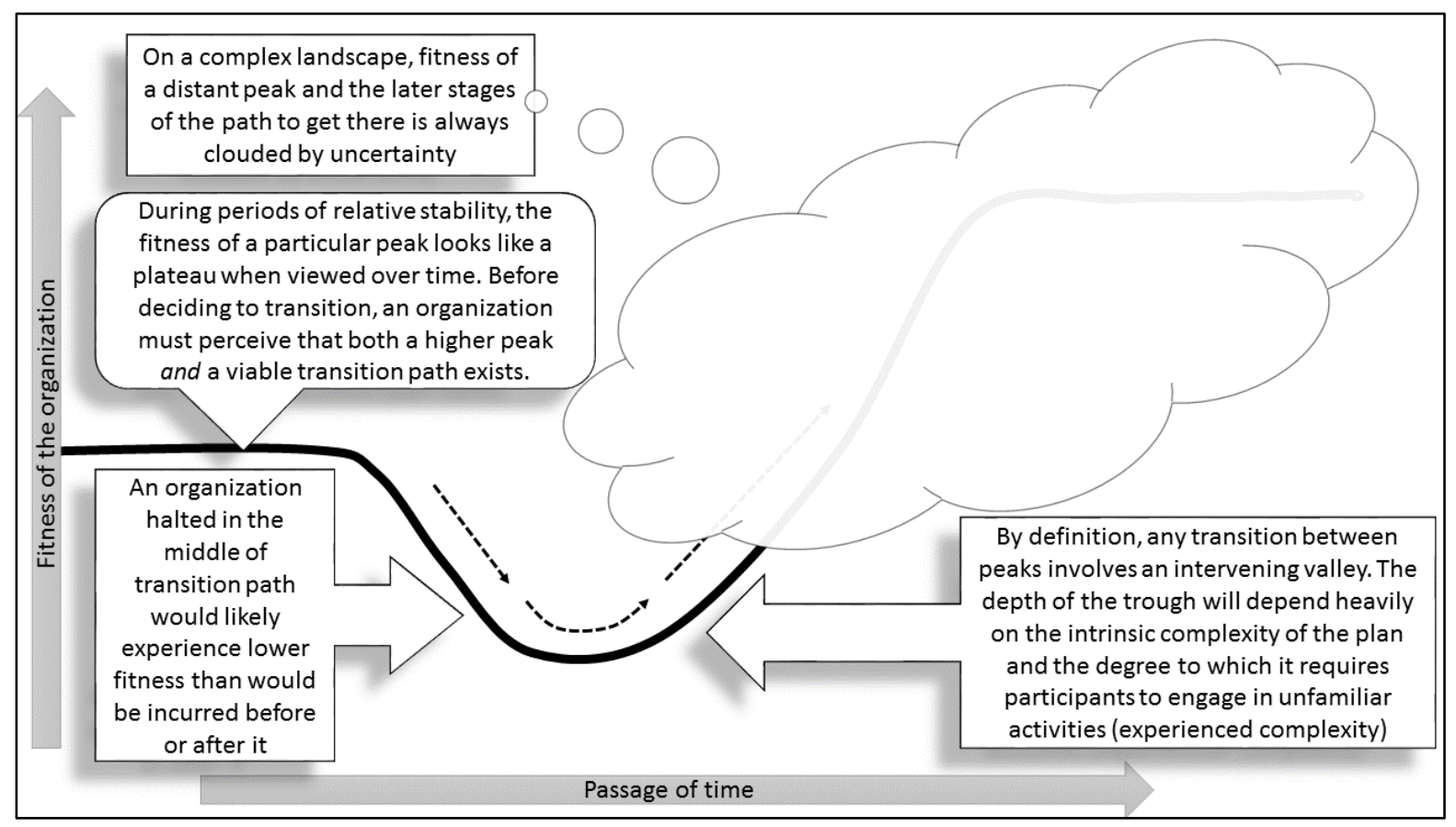

Figure 2: A model of transitions from an initial fitness peak to a target peak

The most important feature of "long jumps" as they are described in the literature is the degree to which they represent a transition to a state whose fitness is very uncertain. To achieve the same intent, we propose a construct that captures the relative density of state-sets of highly uncertain setfitness within the landscape. The associated uncertainty could, for example, be a consequence of a particular state set having no occupants (in which case the denominator of the realized fitness function would be 0 ), very low occupancy, or rapidly changing realized fitness values. We refer to this characteristic as maturity. We can think of certain changes - such as the discovery of a new technology or a major deregulation-as opening up a previously inaccessible state-set within the broader fitness landscape. At the point in time when the disruption occurs, the newly accessible landscape remains unexplored, and the fitness of state-sets within the landscape is likely to be highly uncertain. This would be an example of an immature landscape. As organizations begin to occupy the region, the approximate shape of the landscape's fitness function begins to resolve itself and experience reduces the level of uncertainty. At the point where the fitness function for the region is largely understood, we refer to the region as mature, recognizing the complete elimination of uncertainty in set-fitness is unachievable. With maturation, we would expect an organization's perspective on the landscape to change from a broad one, emphasizing exploration, to a more targeted, narrow focus emphasizing the exploitation of a local, well-understood region of the landscape (Levy, 2000). In an NK landscape implementation, the rugged landscape structure used by Kauffman (1993), this would correspond to locking down specific state attributes, which has the effect of reducing the number of combinations of unlocked attributes that are open for the organization to consider. Effectively, doing so shrinks the state-set available to the organization.

Simple landscapes will tend to mature more rapidly than extrinsically complex landscapes. In such cases, a simple static-fitness function is compact and can be readily estimated by employing a process of incrementally testing individual attributes. Because the set-fitness of a state-set is heavily influenced by the movement of entities into and out of the state-set, a landscape cannot mature until it is relatively stable with respect to peak occupancy. We would also expect a landscape's maturity to be heavily influenced by external factors. For example, rapidly advancing technologies, the frequent identification of new markets, and continuously changing regulations might lead an industry to mature slowly, if ever. Similarly, forces that artificially limit the landscape to certain state-sets could lead 
to rapid maturation and corresponding narrow participant focus without necessarily impacting the survivability of existing participants. This could be the case in a highly restrictive regulatory environment or one with strong protections for indispensable intellectual property. For example, companies like Xerox and Polaroid used patent protection to keep their landscapes very stable. Once those protections expired (Xerox) or were overtaken by new technologies (Polaroid), the landscapes became very dynamic. We further conjecture that a major unexpected disruptive event, e.g., Taleb's (2007) black swan, could move a mature landscape back towards immature. For example, deregulation of the U.S. airline industry in 1978 transformed a previously stable industry in ways that none of the participants had predicted. Figure 3 summarizes the expected pattern of maturation and focus.

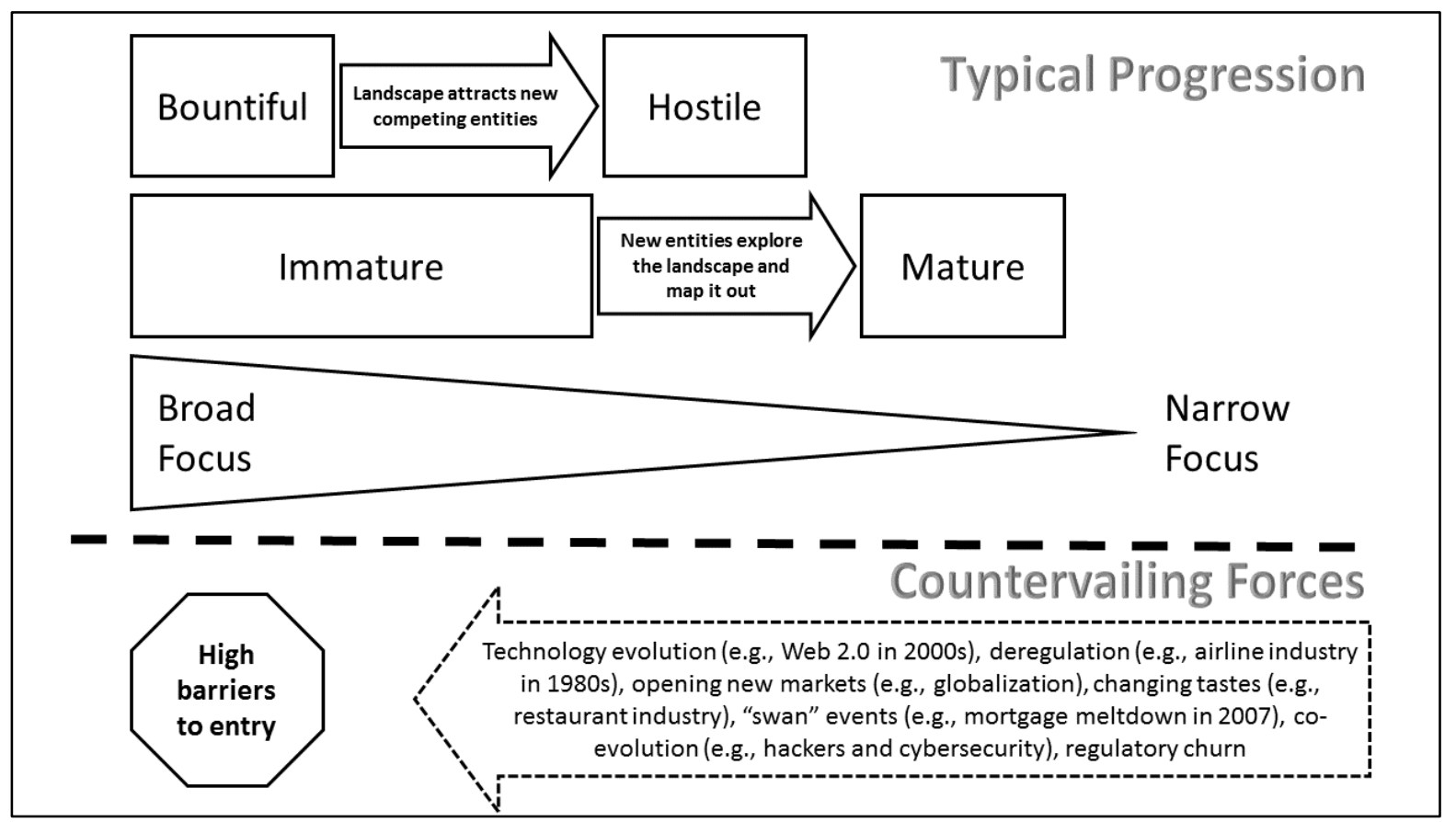

Figure 3: Forces leading to landscape maturation and countervailing forces

Implied within the bountiful to hostile transformation of Figure 3 is the assumption that landscapes are ultimately capacity-constrained with respect to occupancy. We would, therefore, expect that bountiful environments will generally tend to be capacity insensitive in their early stages, whereas hostile environments will tend to be capacity sensitive as growth in the number of participants reduces the fitness levels of existing participants. Not all landscapes will experience this behavior, however. As noted previously, landscapes subject to a network effect (Uzzi, 1996, p. 694) could behave quite differently, with increasing occupancy improving fitness.

\section{FITNESS PROXIES}

Despite being unknowable, set-fitness is a very important long-term construct for entities on a landscape. When applied to a single organization, it captures the likelihood that it will survive each period. That would certainly be a value that the organization and its stakeholders would want to know. Past realized fitness will not necessarily provide definitive guidance-recall the example of the turkey before Thanksgiving. Organizations and individuals would therefore be motivated to develop an estimate of fitness that can support decision-making. We refer to such estimates of fitness as fitness proxies.

Some proxies may serve as complete substitutes for fitness. For example, one widely cited view of the firm treats shareholder value as the sole source of a company's fitness (Jensen, 2001). Other proxies may be perceived as values that contribute directly and independently to overall fitness. For 
example, a company might seek to increase surveyed customer satisfaction ratings on the assumption that it is always better to have satisfied customers than dissatisfied customers, all other things being equal. Many plausible proxies have a social dimension, which is to say that they depend upon the behaviors and performance of other stakeholders, competitors, or observers. Examples include expert rankings as in Consumer Reports product ratings or published university rankings, popularity counts such as YouTube views, consensus ratings such as Netflix star ratings, observed behaviors of competitor or other organizations, and benchmarks (Gill \& Mullarkey, 2017).

For an agent, a good fitness proxy will be a value or construct expected to contribute to fitness without strong interactions with other in-use proxies. A good fitness proxy will also:

- Provide an ordinal or cardinal value that can be used for choosing between alternative possible states.

- Respond rapidly to state changes, providing timely feedback.

- Be observed or acquired at low or no cost.

- Be influenced by the organization's behavior.

- Behave in ways that can be modeled, allowing the organization to consider how alternative actions are likely to impact the proxy's value.

Assuming a rugged landscape, we also can characterize environments with our two previously described attributes:

1. Survivability (hostile to bountiful), reflecting the mean set-fitness of the accessible states of the landscape.

2. Maturity (immature to mature), reflecting the degree to which the approximate fitness of states on the landscape is known.

While it might be possible to characterize simple landscapes (i.e., decomposable landscapes with a single peak) using these dimensions as well, we doubt they would be very useful. Maturity, in particular, is likely to be achieved very quickly in these landscapes since the impact of individual attributes can be determined separately.

\section{INFORMING SOURCES}

The absence of immediate feedback with respect to set-fitness means that considerable effort is required to interpret and make sense of the agent's own experiences. As part of this sense-making process, agents will have a strong motivation to act as informing system clients and to seek out other agents that can act as informers. These informers can help clients address questions such as:

- What fitness proxies should the client adopt?

- How should the client choose the direction of the next transition?

- When does it make sense for a client to attempt a transition towards a new peak?

Naturally, the number of potential informers to which a client could attend is huge. For our purposes, then, we will classify informers according to two different attributes:

1. Accessibility: External vs. Internal

2. Role: The role the informing source plays in the environment. This can be further broken down into two dimensions

a. Impacted: The degree to which the informer is impacted by changes to the fitness landscape

b. Impacting: The degree to which the informer can impact the fitness landscape

We now describe briefly these two attributes. 


\section{ACCESSIBILITY: EXTERNAL VS. INTERNAL}

The accessibility attribute refers to the degree to which the informing source to be consulted is found within the client's existing informing system. Internal sources are those that already contribute to the client's existing informing processes, normally in interactive fashion. Where the client is an individual, examples of these might include:

- Past experience

- Friends and family

- Close colleagues at work

- The client's managers and individuals the client manages

Where the client is an organization, examples of internal sources might include:

- Information within the organization's information systems

- Current stakeholders (e.g., customers, employees, directors, suppliers, investors)

- Professionals with whom the organization has a long-standing relationship (e.g., lawyers, accountants)

External sources (1) exist outside the client's existing informing systems and (2) tend to be less interactive in nature. Where the client is an individual, examples of external sources might include:

- Books, magazines, newspapers and other publications

- Websites and broadcasts

- Public data sites

- Help desks

- Strangers

- Salespeople

Where the client is an organization, external sources might include:

- Industry databases

- Competing firms

- Regulators

- Trade associations

- External auditors

The internal vs. external distinction is a matter of degree rather than binary in nature. For example, the first time a client consults a lawyer, the lawyer is likely to be characterized as an external source. If an ongoing relationship is established — such as a retainer-and consultations occur frequently, the relationship will increasingly become internal to the client's informing system. In order to achieve a sale, a salesperson will frequently attempt to achieve a high level of interaction with the client in order to be perceived as less of an outsider. There may also be situations where the decision is made to exclude an informer that formerly held a position within an informing system. For example, two researchers may have collaborated on projects in the past. In the event that they find themselves competing for the same grant, however, they may be reluctant to share proposals or insights gained from private discussions with a program director, despite maintaining an otherwise collegial relationship.

\section{Role: PEER, PARTICIPANT, INFLUENCER, FACILITATOR, AND OBSERVER}

The role attribute specifies the degree to which the informing source actively participates in the landscape facing the client. For this attribute, however, there are five possible roles:

- Peer: Impacted/Impacting. The informer participates in the same landscape as the client in a manner paralleling that of the client. For example, in a classroom setting, students taking the 
same class would be peers. In an industry setting, organizations that are direct competitors would similarly be classified as peers.

- Participant: Impacted/Not Impacting. The informer participates in the region of the landscape that is of interest and is impacted by the factors that determine the landscape and by the client's positioning, but is not necessarily subject to the same fitness function as the client. For example, in the classroom setting with the client as a student, an auditor might be considered a participant. In an industry setting, a client's minor suppliers and customers might fall into this category.

- Facilitator: Not Impacted/Impacting. The informer can impact the landscape and its corresponding fitness function without participating directly in the landscape and being impacted by its fitness. In a classroom setting the instructor would be classified as a facilitator. In an industry setting, regulators would be similarly positioned.

- Observer. Not Impacted/Not Impacting. The informer's information derives from observing the overall landscape or region of the landscape that is of interest without impacting, or being directly impacted by, the landscape's fitness function.

- Influencer: Somewhat Impacted/Somewhat Impacting. The informer, while not experiencing fitness in the same manner as the client, is nevertheless impacted by the client's fitness and may be able to exert some influence on the client, particularly with respect to the client's positioning on the landscape. In the classroom setting, this might include individuals such as the client's parents and partners. In an industry setting, this might include the client's investors, major suppliers and major customers.

Another way to look at these classifications is in terms of the degree to which the informer can impact and is impacted by the fitness function that is experienced by the client. This is summarized in Table 1.

Table 1: Alternative Informer Roles

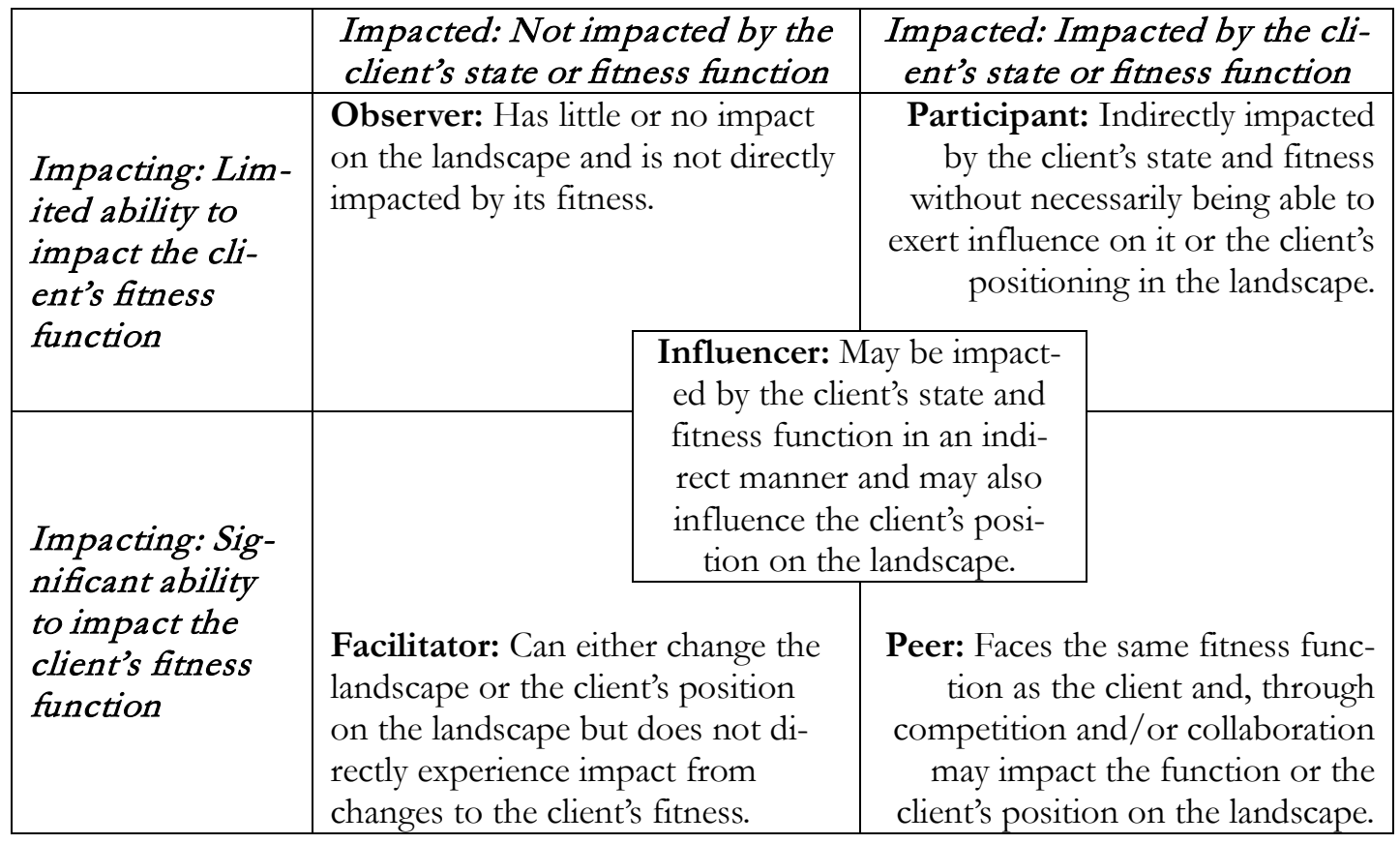

A simple illustration of how the distinction might break down can be found in a sporting event. From a player's perspective, the competing athletes would be classified as peers. Coaches would be characterized as influencers - they are impacted by the landscape but not necessarily in the same way as players, and they also have the ability to impact how players are positioned on the landscape. Par- 
ticipants might include broadcasters and vendors, who will likely be influenced by the nature of play, but may not be able to directly influence it. The referees or umpires would be classified as facilitators; while they set and enforce the rules, their actions do not (or should not) be impacted by who wins or loses. The fans would be observers.

Much like the internal vs. external distinction, role classification will be fuzzy in many instances. Consider, for example, the client's decision of whether or not to place a particular ethnic restaurant-we will use a fast casual Thai restaurant franchise for this example-in a specific shopping center. Examples of informing sources likely to be classified as observers might include:

- Broad insights from restaurant consultants

- Discussions with random individuals in the mall

Consultants and mall patrons are observers due to the low likelihood they will be directly involved in determining the viability of the proposed restaurant (i.e., its fitness) or directly impacted by it. Informing sources falling into the participant category might include:

- Interviews with customers of other fast casual ethnic restaurants in the same mall, since these individuals would seem likely to be potential customers.

- Conversations with owners of Thai restaurants in the surrounding area, since the likelihood that we may be attracting customers away from them is high.

Informing sources that might exist somewhere in the middle of the participant-observer continuum-where future participation in the local landscape is possible but is likely to be limited-might include:

- Insights from the owners of sit-down restaurants in the same mall with more traditional cuisine. Although they participate in the same mall area, the different types of food they serve suggests their fitness function may be very different from that of the proposed restaurant.

A particularly interesting case is that of conversations with the franchisor, the company from whom we would acquire the rights to set up our restaurant. Since the franchisor sets the rules that must be followed by franchisees, it operates in a facilitator role. Since most franchisors also establish a certain number of company-owned units in order to demonstrate the viability of the franchise and acquire information (e.g., test new menu items, store layouts), they can also inform from a participant's perspective.

On a highly rugged landscape, it is reasonable to assume the more an informer's knowledge is based upon direct participation, the more likely the information will apply to the client's situation. This needs to be weighed against the potential impact of interacting with self-similar peers operating on the same landscape-who may well be competitors on the same landscape. Such potential competition can both increase the risk that self-similar peers will be unwilling to share information, may be motivated to misinform, or may gain competitive insights from the interaction that are more beneficial to the informer than to the original client that initiated the interaction.

\section{TOWARDS A THEORY FOR PRIORITIZING INFORMING SOURCES ON RUGGED LANDSCAPES}

Since set-fitness cannot be known, as explained previously, clients seeking to achieve higher fitness will need to rely on both their own experimentation and fitness proxies. An interesting question, therefore, is where should such proxies come from? In particular, we propose that two characteristics of the landscape — survivability and maturity — may exert a significant influence on the priorities that clients place on different informing sources.

We begin by assuming that clients are intelligent agents that occupy a landscape with other agents. These other agents can consist of both other clients and agents engaging in alternative roles, such as 
regulators. The goal of a client agent is to acquire information so as to maximize fitness. In doing so, it will particularly need to:

- Reduce the overall uncertainty associated with fitness on the environment.

- Minimize conditions where the behaviors of other agents on the landscape (facilitators, influencers, peers) reduce the fitness of desirable positions through rule-making, competition, or bargaining power.

- Maximize conditions where the behaviors of other agents on the landscape increase the fitness of desirable positions, for example through rule-making, uncertainty reduction, or bargaining power.

- Prioritize which agents (a) they will seek to be informed by, (b) they will seek to inform, or (c) with whom they will exchange information to maintain or increase fitness.

We also need to consider two scenarios: one in which a client already participates in the existing landscape, and one where a client seeks to enter the landscape.

\section{EXISTING LANDSCAPE CLIENTS}

For clients participating in a landscape, we propose priorities will be set along the lines summarized in Table 2.

Table 2: Expected Informing Priorities According to Rugged Landscape Classification for Existing Client

\begin{tabular}{|c|c|c|}
\hline & Hostile & Bountiful \\
\hline Immature & $\begin{array}{l}\text { High Priority } \\
\text { - External Agents } \\
\text { - Influencers - Exchange } \\
\text { - Facilitators - Exchange } \\
\text { Low priority } \\
\text { - Participants - Informed By }\end{array}$ & $\begin{array}{l}\text { High Priority: } \\
\text { - External Agents } \\
\text { - Peers - Exchange } \\
\text { - Facilitators - Exchange } \\
\text { - Influencers - Exchange } \\
\text { Lower Priority } \\
\text { - Participants - Exchange }\end{array}$ \\
\hline Mature & $\begin{array}{l}\text { High Priority } \\
\text { - Internal Agents } \\
\text { Lower Priority } \\
\text { - Facilitators - Informed by } \\
\text { - Influencers - Exchange } \\
\text { - Observers - Informed by }\end{array}$ & $\begin{array}{l}\text { High Priority } \\
\text { - Facilitators - Inform } \\
\text { - Internal Agents } \\
\text { Lower Priority } \\
\text { - Peers - Exchange } \\
\text { - Observers - Informed by }\end{array}$ \\
\hline
\end{tabular}

The locations assigned to the Table 1 informers in Table 2 are driven by the following general assumptions:

- Observers: For immature, rugged landscapes (top rows), observers are unlikely to provide very useful insights. These landscapes tend to have characteristics that limit the usefulness of general expertise (Shanteau, 1992; Taleb, 2007). For mature landscapes, highly specialized expertise may provide some insights.

- Peers \& Influencers: Because both tend to impacted by the same landscape as the client, these agents are unlikely to serve as informers unless they are informed in the process. Thus, we assume they always participate in exchange relationships.

- Internal sources will likely dominate mature environments - since participation in the landscape builds knowledge of its contours, which do not change much over time (being mature). 
- External sources will be critical in immature environments since participants in the landscape will not yet have the knowledge to create a reliable map_and such a map may not be possible owing to its dynamic nature.

The specific quadrant assignments are based on the following analysis.

\section{Immature-Bountiful}

This quadrant would be the starting point for many attractive, emerging landscapes. Because it is immature, reducing uncertainty as quickly as possible is likely to be a top priority. With little information available from past experience, external sources are likely to be a high priority. Because it is bountiful, competition from other peers is of limited concern; this might be the case in landscapes subject to network effects. On a highly rugged landscape, the more similar two peers are to each other the more likely a change in behavior in one will produce a similar change in fitness for the other (Gill, 2012). Thus, exchanging information with peers is likely to prove a very effective informing source. The other very effective source would be facilitators responsible for controlling elements of the landscape; because of its immaturity, they are likely to be very responsive to feedback, indicating an information exchange. We also expect clients to willingly exchange information with influencers and participants, again because stimulating competition would not be of great concern.

On an individual level, a student client enrolling in a new course dealing with a topic not previously taught or employing an unfamiliar pedagogy might be an example of such a landscape. The landscape would be established by the course syllabus. The instructor, in the role of facilitator, would be a good source of information but would also be seeking feedback owing to the novelty of the course. The bountiful nature of the environment, such as a course design with relaxed grading, would likely encourage student peers to exchange information about assignments and projects. The immaturity of the environment would suggest that highly structured information sources from participants, such as textbooks, would not be available and thus make these lower priorities. Other influencers and participants would also be secondary sources. The nature of the landscape would suggest there would be reason to exchange information with these sources freely.

A rapidly emerging high tech industry might be an organizational example. Peers in these industries often cluster together, sharing information freely during the early stages. Organizations in facilitating roles may or may not exist in such an environment; where they do, clients would exchange information freely with them. Influencers (venture capital sources, major customers, and suppliers) would also be useful sources. Participants would likely be less knowledgeable in such a context, but may serve as useful lower priority sources.

\section{Immature-Hostile}

This type of environment would be similar to the immature-bountiful environment except that peers would likely be excluded from informing channels for fear of attracting competition. That would raise the importance of exchanging information with influencers and facilitators (where the latter exist). To avoid attracting competitors, however, clients might be reticent to over-share with participants who might be attracted to the landscape.

In the new course example, if the instructor indicated that the course was to be graded on a strict curve, students' attitudes towards sharing work with peers could change significantly because they would be competitors rather than collaborators. Similarly, students might not seek to attract new peers from the participant group (which would include students considering registering), particularly if those peers were seen as strong potential competitors.

In the industry example, once a landscape becomes highly competitive, peers would be much less likely to share openly with each other. They might also mine participant information sources-such as employees hired away from competitors-but would not necessarily be eager to share with them. 


\section{Mature-Hostile}

In the mature-hostile environment, the client is fully familiar with the landscape and is therefore likely to rely on internal sources_-such as past performance data-rather than seeking out external sources. In terms of secondary sources, because mature-hostile environments are not likely to be attractive to the entry of new peers, the client would probably want to monitor facilitators but would not necessarily be that concerned about influencing them. Exchanging information with influencers and information provided by informed observers could also be beneficial.

At the individual level, a client working in a stable job in competition with other employees in the same position would probably avoid too much sharing with other peers. He or she would likely keep an eye on job performance metrics to ensure performance was acceptable. Secondarily, keeping an eye on company policy for changes (informing from facilitators) would be warranted and he or she would likely be open to information exchange from influencers (such as supervisors).

At the organizational level, accounting, financial, and production data could be used to monitor and tune performance (internal sources). Secondarily, the firm would likely keep an eye on the regulatory environment and be willing to exchange information with important influencers (e.g., major customers, suppliers, bankers). We would also expect that the client would be receptive to data from external observers, such as industry reports and research.

\section{Mature-Bountiful}

Per Figure 3, we would not anticipate that the mature-bountiful landscape would last very long under normal circumstances. The exception to this would be in cases where facilitators created a set of rules that benefit existing peers and also serve to prevent the entry of existing peers. For this reason, we propose that informing facilitators would be a top priority. While informing describes the flow, the "information" being conveyed would not necessarily represent an accurate depiction of the landscape. Instead, it would more likely be framed in a manner that benefits the client.

Because any peers in the environment would necessarily be benefiting from the same rules that benefit the client, the client would potentially be open to exchange of information with those peers. Similarly, the client would likely be receptive to informing from observers, although the expected benefits would not necessarily be great. Free exchange of information with influencers might also be avoided, for reasons similar to those not freely exchanging information with facilitators.

At the individual level, workers whose secure jobs are paying well above market rates might well support regulation such as licensing rules and job protections to prevent new individuals from entering the field. They might also band together with peers (e.g., form a union) to achieve the same effect. They would not, however, invite outside inspection of their work that would bring attention to it, especially from influencers knowledgeable enough to understand its nature.

At the organizational level, clients may lobby for legislation that serves to keep new entrants out, perhaps banding together with other participants in the same industry to increase the impact of the lobbying. They might also keep an eye on the macroeconomic data gathered by the same industry association.

\section{NEWLANDSCAPE ENTRANTS}

The proposed conceptual scheme would differ slightly for clients seeking to enter a landscape as opposed to those already on the landscape. Since the immature-bountiful combination is expected to be relatively welcoming to new participants, the most beneficial informing sources seem unlikely to change. For the mature-bountiful combination, however, reliance on external sources will be required, since internal sources will not be operating on the landscape. Moreover, peer exchange is likely to be precluded since the new entrant has little to share. Most significantly, new entrant clients will 
likely seek to take an active role in informing of facilitators with the goal of providing information supportive of breaking down barriers versus building and maintaining such barriers to entry.

For immature-hostile environments, the informing sources are also likely to be similar for existing participants and new entrants. Because new entrants will likely be seeking alternative peaks to those already occupied, exchange of information with participants is likely to play a larger role since influencers will likely have relationships with existing peers.

Entry into mature-hostile environments is likely to be very difficult, since existing peers are well established, the overall environment is not inviting, and entrants new to the landscape are likely to rely heavily on external sources since they have not acquired the internal sources that would come from prior participation in the landscape. The approach most likely to succeed would be to find a region of the environment that is not well understood — an immature pocket. To find such a pocket, information from observers and exchange of information with influencers and facilitators would be the best sources.

Disruptive innovation (Christensen, 2013) represents an example of this type of process. In that scenario, an existing occupant within an industry (e.g., film photography) serves a stable base of customers and focuses on its needs exclusively. A new entrant - the disrupter-focuses on an entirely new customer base (e.g., computer enthusiasts) with a new technology (e.g., digital photography) or approach that is usually inferior to the existing technology (e.g., film) but that also has the potential for rapid evolution. Even though the original organization may be aware of the new approach (e.g., Kodak had many of the original digital photography patents and began selling digital cameras well before they became ubiquitous), it continues to focus on its main customers, since they are the main source of its profits. At some point in time, however, the new technology exceeds the original technology in both quality and affordability. At that point in time, the customers of the original company depart en-masse (and its fitness goes to zero).

\section{CONCLUSIONS}

When facing a complex environment, how should we prioritize different sources of information? This is a question that has not been addressed within informing science, yet it is one of importance to clients with limited resources available to attend to all possible sources. In this paper, we have proposed an initial conceptual scheme that is built around:

- Classifying complex environments

- Classifying alternative types of informers

- Mapping different types of informers to differing environmental characteristics

While we are not yet ready to describe our analysis as a full-fledged theory, we nevertheless believe the analysis we presented will be a useful starting point in the process of understanding the fit between informer and client.

In describing complex environments, we begin with the concept of a fitness landscape. Such landscapes map the attributes of the client to a likelihood of period to period survival. For the purposes of classifying these environments, we find two dimensions to be particularly relevant:

1. Survivability: How difficult is it for a client to persist in the environment over time? Some rugged landscapes are bountiful - meaning that clients existing on the landscape tend to survive for a very long time. At the other extreme of the continuum is hostile, where clients experience a continual period-to-period battle for survival.

2. Maturity: How difficult is it for clients to predict the fitness of a particular state before occupying it. In immature environments, the fitness of most states is shrouded in uncertainty. In mature environments, the fitness of most states can be predicted reliably. Landscapes may al- 
so contain a mixture of regions, some areas being mature, some being immature-perhaps having been opened up by new technologies or changes in rules or regulation.

In classifying landscapes, we also point out that these dimensions are likely to be dynamic. Decisions made by clients in one time period impact the complexity of the landscape (and thus additional decisions) in subsequent periods. Over time, we expect landscapes to move from bountiful to hostile and from immature to mature. External forces, such as evolving technologies and changing regulations can alter this progression, however.

For informers, a classification approach has also been taken. Accessibility describes where the informer is coming from. External informers come from outside of the client's normal informing system; internal informers already exist within the client's system. Role describes the informer's position relative to the landscape. Impacting informers have the ability to impact the fitness landscape that impacts the client-although they may not experience the same fitness function; non-impacting informers have limited or no ability to impact the landscape. Impacted informers experience fitness that depends on all or many of the same attributes that determine the client's fitness; non-impacted informers are not subject to the same or similar influences. Using these attributes, we assigned names to a series of different types of informers: facilitators (impacting/non-impacted), observers (nonimpacting/non-impacted), peers (impacting/impacted), and participants (non-impacting/impacted). An additional classification, influencers, was defined to capture intermediate values of both attributes.

In broad terms, our conceptual scheme was built around the following premises:

- Clients facing immature environments will tend to rely heavily on external sources; clients operating in mature environments will rely more heavily on internal sources. Clients seeking entry into an environment will nearly always be forced to rely heavily on external sources.

- Clients in bountiful environments will tend to be much more likely to exchange information with peers than those in hostile environments.

- In mature environments, facilitators will play a critical role; much less so in immature environments.

- Informers impacted by the client's landscape will normally be willing to offer information only in exchange for information from the client; this will be less true for informers not impacted.

We believe that with empirical validation, the conceptual scheme that we have proposed has the potential to evolve into a theory of informing sources. Future work in this area can move in several directions. Significant opportunities exist in refining the nature and classifying various fitness proxies used to estimate the ruggedness of the decision landscape. Also, development of proxy measurements to help determine the hostile nature and maturity status of the landscape can be pursued. Of course, additional prospects for future research are in determining appropriate forms of fitness functions for differing degrees of landscape hostility and maturity. Finally, we believe there may be beneficial opportunities to expand the set of informer roles that we have proposed.

\section{REFERENCES}

Billinger, S., Stieglitz, N., \& Schumacher, T. R. (2014). Search on rugged landscapes: An experimental study. Organization Science, 25(1), 93-108. https://doi.org/10.1287/orsc.2013.0829

Christensen, C. (2013). The innovator's dilemma: when new technologies cause great firms to fail. Boston, MA: Harvard Business Review Press.

Cohen, E. B. (1997). IS as an evolving field. In N. Callaos, C. M. Khoong, \& E. Cohen (Eds.), Proceedings of the World Multiconference on Systemics, Cybernetics, and Informatics, Caracas, Venezuela, July 7-11. 
Cohen, E.B. (1999). Reconceptualizing information systems as a field of the discipline informing science: From ugly duckling to swan. Journal of Computing and Information Technology, 7(3), 213-219.

Gill, T. G. (2012). Informing on a rugged landscape: Homophily versus expertise. Informing Science: The International Journal of an Emerging Transdiscipline, 15, 49-91. https://doi.org/10.28945/1560

Gill, T. G. (2015). Informing science, volume 1: Concepts and systems, Santa Rosa, CA: Informing Science Press.

Gill, T. G., \& Mullarkey, M. (2017). Fitness, extrinsic complexity, and informing science. Informing Science: The International Journal of an Emerging Transdiscipline, 20, 37-61. https://doi.org/10.28945/3668

Jensen, M. C. (2001). Value maximization, stakeholder theory, and the corporate objective function. Journal of Applied Corporate Finance, 14(3), 8-21. https://doi.org/10.1111/j.1745-6622.2001.tb00434.x

Kauffman, S. A. (1993). The origins of order. Oxford, UK: Oxford University Press.

Levy, D. L. (2000). Applications and limitations of complexity theory in organization theory and strategy. Public Administration and Public Policy, 79, 67-88.

Merali, Y., \& Allen, P. (2011). Complexity and systems thinking. The SAGE handbook of complexity and management (pp. 31-52). Los Angeles, CA: Sage.

Porter, M. E. (2008). The five competitive forces that shape strategy. Harvard Business Review, 86(1), 25-40.

Rogers, E.M. (2003). Diffusion of innovations (5th ed.). New York, NY: Free Press.

Shanteau, J. (1992), Competence in experts: The role of task characteristics. Organizational Behavior and Human Decision Processes, 53, 252-266. https://doi.org/10.1016/0749-5978(92)90064-E

Taleb, N. N. (2007). The black swan: The impact of the bighly improbable (2nd ed.). New York: Random House.

Uzzi, B. (1996). The sources and consequences of embeddedness for the economic performance of organizations: The network effect. American Sociological Review, 61(4), 674-698. https://doi.org/10.2307/2096399

\section{BIOGRAPHIES}

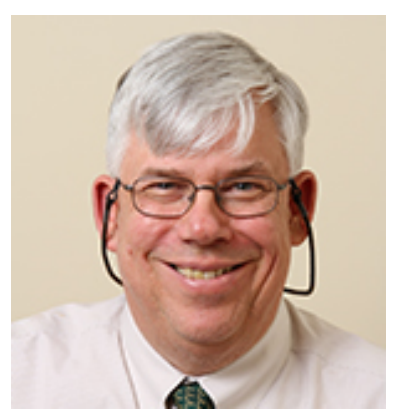

Grandon Gill is a professor in the Information Systems and Decision Sciences department of the University of South Florida. He is also the Academic Director of the Doctor of Business Administration program at the Muma College of Business. He is Editor-in-Chief of Informing Science: The International Journal of an Emerging Transdiscipline and the Journal of IT Education: Discussion Cases, also serving as a Governor and Fellow of the Informing Science Institute. In 2014, he was the inaugural recipient of the Zbigniew Gackowski Award for contributions to informing science research.

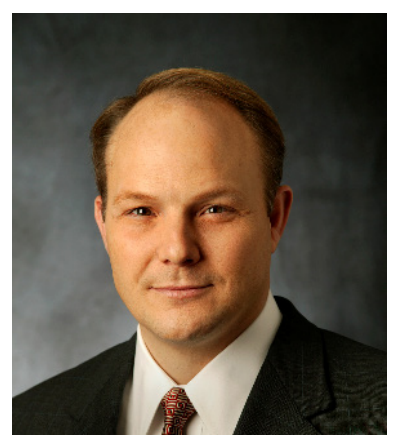

Matt Mullarkey is the Director, Doctor of Business Administration Program at the USF MUMA College of Business and an Instructor in the Information Systems and Decision Sciences department at the University of South Florida. He holds a Ph.D. in Business Administration (Information Systems) from USF, a BS, Engineering, from the United States Military Academy, an MS, Systems Management, from the University of Southern California, and an MBA from the Moore Business School, University of South Carolina. Matt has more than 25 years of experience as President, CEO, COO, and SVP in the automotive, applied materials and medical device industries with P\&L responsibilities globally. His principal research interest are the impact of social networking in and between organizations, monetization of companies' data streams, and applying the case method to MIS and MBA education. He is an editor for the Journal of IT Education: Discussion Cases. 


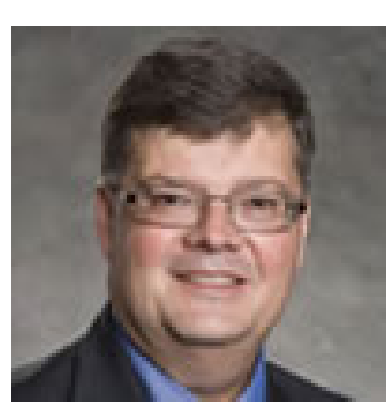

Ron Satterfield is an instructor in the Information Systems Decision Sciences Department of the USF Muma College of Business, teaching courses in statistics, operations management, and process improvement. He teaches these courses in the USF undergraduate, MBA, Executive MBA, MS in Business Analytics and Information Systems, and Doctorate of Business Administration programs. He earned a PhD in Operations Management from the Kelley School of Business at Indiana University and an MBA and BA in operations management from Eastern Illinois University. He is also a board member and chair of the IT Committee for the USF Federal Credit Union. 